\title{
Recoiling Black Holes: Electromagnetic Signatures, Candidates, and Astrophysical Implications
}

\author{
S. Komossa ${ }^{1,2,3}$ \\ ${ }^{1}$ Fakultät für Physik, Technische Universität München, James Franck Straße 1/I, 85748 Garching, Germany \\ ${ }^{2}$ Excellence Cluster Universe, TUM, Boltzmannstraße 2, 85748 Garching, Germany \\ ${ }^{3}$ Max-Planck-Institut für Plasmaphysik, Boltzmannstraße 2, 85748 Garching, Germany
}

Correspondence should be addressed to S. Komossa, stefanie.komossa@gmx.de

Received 11 November 2011; Accepted 5 January 2012

Academic Editor: Francesca Civano

Copyright $(2012$ S. Komossa. This is an open access article distributed under the Creative Commons Attribution License, which permits unrestricted use, distribution, and reproduction in any medium, provided the original work is properly cited.

\begin{abstract}
Supermassive black holes (SMBHs) may not always reside right at the centers of their host galaxies. This is a prediction of numerical relativity simulations, which imply that the newly formed single $\mathrm{SMBH}$, after binary coalescence in a galaxy merger, can receive kick velocities up to several $1000 \mathrm{~km} / \mathrm{s}$ due to anisotropic emission of gravitational waves. Long-lived oscillations of the SMBHs in galaxy cores, and in rare cases even SMBH ejections from their host galaxies, are the consequence. Observationally, accreting recoiling SMBHs would appear as quasars spatially and/or kinematically offset from their host galaxies. The presence of the "kicks" has a wide range of astrophysical implications which only now are beginning to be explored, including consequences for black hole and galaxy assembly at the epoch of structure formation, black hole feeding, and unified models of active galactic nuclei (AGN). Here, we review the observational signatures of recoiling SMBHs and the properties of the first candidates which have emerged, including follow-up studies of the candidate recoiling SMBH of SDSSJ092712.65+294344.0.
\end{abstract}

\section{Introduction}

Interaction and merging of galaxies occurs frequently throughout the history of the universe. If both galaxies do harbor SMBHs, binaries will inevitably form [1]. Galaxy mergers are believed to be the sites of major black hole growth, and an active search for SMBH pairs and binaries of wide and small separations is currently ongoing (see [2] for a review of electromagnetic signatures). When the two SMBHs ultimately coalesce, they are a source of strong gravitational waves. These are emitted anisotropically during coalescence and carry away linear momentum (e.g., [3]). As a result, the newly formed single SMBH recoils. Configurations of coalescing black holes can lead to kick velocities up to several thousand $\mathrm{km} / \mathrm{s}$ (e.g., [4-16]; review by [17]). In the initial computations, kick velocity was highest for maximally spinning equal-mass black hole binaries with antialigned spins in the orbital plane ("superkicks"). More recently, based on a new recoil formula, Lousto and Zlochower [18] have estimated that recoil velocities up to $5000 \mathrm{~km} / \mathrm{s}$ can be reached in configurations with spins partially aligned with the orbital angular momentum. In unbound encounters (not likely to occur in astrophysical environments), the kick velocity can exceed $15000 \mathrm{~km} / \mathrm{s}[19,20]$.

After the kick, the recoiling SMBH will oscillate about the core of its host galaxy [21, 22] or will even escape, if its kick velocity exceeds the escape velocity of its host. In a "typical," gas-poor galaxy, a black hole kick velocity of $500 \mathrm{~km} / \mathrm{s}$ will result in an initial amplitude of $\sim 200 \mathrm{pc}$, and an oscillation timescale of order $10^{7} \mathrm{yrs}$ (Figure 1 of [23]). The kicks, including those large enough to remove SMBHs from their host galaxies, have potentially far-reaching astrophysical consequences, including for SMBH and galaxy assembly and AGN statistics. Upon recoil, the most tightly bound gas will remain bound to the recoiling black hole, and therefore highvelocity kicks imply the existence of interstellar and intergalactic quasars (e.g., [21-30]). Identifying recoiling SMBHs through observations is of great interest. Several key electromagnetic signatures of kicks have been predicted in the last few years, and first candidate recoiling $\mathrm{SMBHs}$ have emerged. This chapter is structured as follows. In Section 2, an overview of the predicted electromagnetic signatures of 
recoiling SMBHs is given. In Section 3 the event frequency is discussed, while Sections 4 and 5 provide a review of the published candidate recoiling SMBHs. Section 6 explores consequences of recoil for unified models of AGN. Section 7 concludes with some astrophysical consequences and important future studies.

\section{Electromagnetic Signatures of Recoiling SMBHs}

2.1. Broad Emission-Line Shifts. After the kick, matter remains bound to the recoiling $\mathrm{SMBH}$ within a region whose radius $r_{\mathrm{k}}$ is given by

$$
r_{\mathrm{k}}=\frac{G M_{\mathrm{BH}}}{v_{\mathrm{k}}^{2}} \approx 0.4\left(\frac{M_{\mathrm{BH}}}{10^{8} \mathrm{M}_{\odot}}\right)\left(\frac{v_{\mathrm{k}}}{10^{3} \mathrm{~km} \mathrm{~s}^{-1}}\right)^{-2} \mathrm{pc},
$$

where $v_{\mathrm{k}}$ is the kick velocity [31]. This region is on the order of the size of the broad line region (BLR) of AGN [32]. The accretion disk and BLR will therefore typically remain bound to the SMBH while the bulk of the host galaxy's narrow-line region (NLR) will remain behind. The accreting recoiling $\mathrm{SMBH}$ will therefore appear as an off-nuclear "quasar" as long as its accretion supply lasts. However, spatial offsets are challenging to detect even with the Hubble Space Telescope (HST) except in the nearby universe. The kinematic Doppler shifts of the broad emission lines are, in principle, easy to measure out to high redshifts. Spectroscopically, recoiling SMBHs will appear as AGN which have their broad emission lines kinematically shifted by up to $\sim 5000 \mathrm{~km} / \mathrm{s}$ with respect to their NLRs.

Bonning et al. [33] suggested several criteria, how to identify a recoiling SMBH spectroscopically. Apart from (1) the kinematic shift of the BLR, a candidate recoiling $\mathrm{SMBH}$ should (2) show symmetric broad line profiles, it should (3) lack an ionization stratification of its narrow emission lines, and it should (4) not show any shift between broad MgII and the broad Balmer lines. (In practice, individual recoil candidates may show some (temporary) deviations from this scheme, or exhibit extra features. For instance, just after recoil, the BLR emission profiles would likely be asymmetric. Feedback trails from partially bound gas and disk winds would produce emission-line signatures at various kinematic shifts between zero and the recoil velocity. Once the SMBH has travelled beyond the extent of the classical NLR of a few kpc extent, low-density "halo" gas would dominate the optical narrow-line spectrum, with emission-line ratios characteristically different from the classical NLR.) One object, the quasar SDSSJ092712.65+294344.0, fulfills all of these four criteria and is therefore an excellent candidate for a recoiling SMBH [34]. It will be further discussed in Section 4, together with several other candidate recoiling BHs. More candidates may hide in large samples of peculiar broad-line emitters recently identified in the Sloan Digital Sky Survey (SDSS; [35]).

2.2. Flaring Accretion Disks. In gas-rich mergers, an accretion disk is likely present, even though the inner part of the disk may only re-form after binary coalescence [27, 36-38]. UV, soft X-ray, and IR flares could result from shocks in the accretion disk surrounding the $\mathrm{SMBH}$ just after recoil, or when the inner disk reforms (e.g., [38-46]). These flares may last $\approx 10^{4}$ yrs and may be detectable in current and future sky surveys.

2.3. Tidal Disruption Flares from Stars Around Recoiling SMBHs. Even in the absence of an accretion disk, ejected SMBHs will always carry a retinue of bound stars. Observable effects related to these stars are therefore perhaps the most universal signature of recoil. As the $\mathrm{SMBH}$ moves through the galaxy, the bound, and unbound, stars are subject to tidal disruption, leading to powerful X-ray flares of quasarlike luminosity $[47,48]$, which would appear off-nuclear or even intergalactic. Komossa and Merritt [49] computed disruption rates for the bound, and the unbound, stellar populations under recoil conditions. In the resonant relaxation regime, they showed that the rates are of order $10^{-6} \mathrm{yr}^{-1}$ for a typical postmerger galaxy (Figure 2 of [49]); smaller than, but comparable to, rates for nonrecoiling SMBHs. At an early phase of recoil, the tidal disruption rate can be much higher, when the $\mathrm{SMBH}$ experiences a full loss cone, and travels through the clumpy core environment of a recent merger [49]. The flare rate may temporarily reach values as high [50] as during the peak of the premerger binary phase [51].

Another signature related to the stars bound to the recoiling SMBH is episodic X-ray emission from accretion due to stellar mass loss. Mass loss provides a reservoir of gas, and therefore also optical emission lines from gas at the recoil velocity even in the initial absence of a gaseous accretion disk. Other consequences include the presence of intergalactic planetary nebulae and supernovae, after the ejected SMBH has left its host galaxy [49].

All these signals would generically be associated with recoiling SMBHs, whether or not the galaxy merger is gas-rich or dry, and whether or not an accretion disk is present initially, and they would continue episodically for a time of $\sim 10$ Gyr [49].

2.4. Hypercompact Stellar Systems. While the "tidal recoil flares" are very luminous and can be detected out to very large distances, the compact system of bound stars itself will be detectable in the nearby universe, and would resemble a globular cluster in total luminosity, but with a much greater velocity dispersion due to the large binding mass $M_{\mathrm{BH}}$ [49]. Merritt et al. [52] worked out the properties of these "hypercompact stellar systems" (HCSSs), and related the structural properties (mass, size, and density profile) of HCSSs to the properties of their host galaxies and to the amplitude of the kick. Since the kick velocity is encoded in the velocity dispersion of the bound stars, future detection of large samples of HCSSs would therefore allow us to determine empirically the kick distribution, and therefore the merger history of galaxies in clusters. Nearby clusters of galaxies are best suited to search for and identify HCSSs, and $~ 100$ of them should be detectable within $2 \mathrm{Mpc}$ of the center of the Virgo cluster [52]. Depending on the merger history of our Milky Way [53], and the merger history of black holes in its globular clusters [54], 100 s of low-mass HCSSs and rogue black holes 
may reside in the halo of our Milky Way, and a search for them is underway [55].

2.5. Other Observable Effects of Recoil. During the long-lived "Phase II" recoil oscillations [22], when the SMBH oscillation amplitude is on the torus scale, the SMBH might efficiently accrete from the dense molecular gas at each turning point, causing repeated flares of radiation [23]. Such flares would locally destroy the dust, while photoionization of the dense surrounding gas would produce a strong emission-line response. Such a signal would not only help in identifying kicks but also could be used as a new probe of the properties of the torus itself.

Other signatures of recoiling SMBHs include effects on the morphology and dynamics of the gaseous disk of the host galaxy [56], their imprints on the hot gas in early-type galaxies [57], accretion from the ISM [58], the possibility of star formation in the wake of the SMBH trajectory [59], and their influence on the jet structures in radio galaxies [30].

\section{The Frequency of Recoiling SMBHs in Astrophysical Environments}

Several factors affect the distribution of SMBH kick velocities and their observability; the system parameters of the $\mathrm{SMBH}$ binary on the one hand (mass ratio, spin magnitudes and spin directions), and the astrophysical environment on the other hand.

The frequency of high-velocity kicks depends on the distribution of mass ratios and spins of the binary SMBHs. In case of random distributions of spin directions, as expected in gas-poor galaxy mergers, the kick formula (e.g., $[4,9,13])$ has been used to predict the kick fraction in dependence of recoil velocity $[4,9,23,60]$. In this case, kicks with velocities larger than $500 \mathrm{~km} / \mathrm{s}$ are relatively common (Figure 1 of [23]). Spin precession further has the consequence that large kicks are deboosted if the angle $\Theta$ between the spin of the more massive $\mathrm{BH}$ and the orbital angular momentum is initially small, while large kicks are boosted, if $\Theta$ is initially large [61].

The other key factor is the astrophysical environment, which determines the spin magnitude (set by the mechanism of SMBH mass growth via random accretion, ordered accretion, or BH-BH merging; [62]) and the timescale of spin alignment with the orbital angular momentum (e.g., [63-65]) in gas-rich galaxy mergers. The latter depends on the rapidity of binary coalescence, the amount of gas accretion before versus after coalescence, the accretion rate, the disk properties (e.g., the viscosity law across the disk), and the mass of the SMBH. While the most massive black holes are more resistant to alignment, the process is generally relatively fast in gas rich environments (timescales of $10^{5}-10^{9} \mathrm{yrs}$ or less) $[66,67]$.

While initial results from numerical relativity have indicated that kick velocities are low in this case, the whole parameter space is still being explored, and Lousto and Zlochower [18] have recently shown that kick velocities up to $5000 \mathrm{~km} / \mathrm{s}$ can be reached in configurations with spins partially aligned with the orbital angular momentum. As a consequence, the likelihood of observing high-velocity recoils in gas-rich environments is significantly higher than in some previous estimates (their Figure 3).

Given the large number of uncertain parameters in estimating the frequency of recoiling SMBHs, identifying them through observations is also important. Ultimately, observations will independently provide the distribution of kick velocities and the properties of the recoiling SMBHs. First candidates have emerged in recent years, and more are likely to come soon, given the number of operating or planned very large spectroscopic and time-domain surveys, like SDSS, LAMOST, LSST, and future X-ray surveys.

\section{Candidate Recoiling SMBHs Identified by Kinematic Signatures}

4.1. SDSSJ092712.65+294344.0 and X-Ray Followups. The quasar SDSSJ092712.65+294344.0 (SDSSJ0927+2943 hereafter) at $z=0.7$ shows all the characteristic optical signatures of a recoiling SMBH [34], which were predicted earlier [33]. Its broad emission lines are shifted by $2650 \mathrm{~km} \mathrm{~s}^{-1}$ with respect to its narrow emission lines, the broad lines are symmetric, the broad MgII line shows the same shift as the broad Balmer lines, and the narrow emission lines lack an ionization stratification as expected if the accreting $\mathrm{SMBH}$ is no longer at the center of the system [34]. (SDSSJ0927+2943 also shows a second system of narrow emission lines with unusual properties when compared with other known quasars, including exceptionally broad Neon emission lines. The origin of these lines is still being explored; the lower-ionization lines are too narrow to have originally been bound to the recoiling SMBH (except in case of projection effects), and their low degree of ionization is not straightforward to understand [34]. A possible reservoir of narrow-line gas at the kick velocity is stellar mass loss, as a consequence of stellar evolution of the stars bound to the recoiling SMBH [49].) Its unique properties make SDSSJ0927+2943 an excellent candidate for a recoiling $\mathrm{SMBH}$.

Two alternative models have been considered in order to explain some (but not all) of the unusual properties of this system; a chance projection, within 1 arcsec, of one or two intrinsically peculiar AGN in a very massive cluster of galaxies $[34,68,69]$, and a close premerger binary SMBH $[70,71]$. However, a rich and massive cluster has not been detected in NIR and X-ray imaging follow-up observations [72], (Komossa et al. 2012, in prep.). Neither was the predicted orbital motion of an SMBH binary detected in spectroscopic followups [68]; see also [73]. This leaves us with the recoil scenario for SDSSJ0927+2943. This scenario is also consistent with the recent measurement of an offset between the QSO and the host galaxy as traced by [OIII] emission [73].

We have obtained an imaging observation of SDSSJ09$27+2943$ with the Chandra X-ray observatory, in order to measure more precisely its X-ray luminosity (than was possible with a serendipitous off-axis ROSAT observation; [34]), and to study the properties of the field around SDSSJ$0927+2943$, including the search for a possible massive cluster of galaxies. We detect point-like X-ray emission from the quasar coincident with the optical position of SDSSJ$0927+2943$. A second X-ray source is present at a distance of 
$\sim 17$ arcsec from SDSSJ0927+2943. This second source coincides with the object SDSSJ092713.8+294336 and contributed approximately $70 \%$ to the ROSAT X-ray emission from the region of SDSSJ0927+2943. Luminous extended X-ray emission from a rich cluster, in the form predicted by Heckman et al. [69], is not present. The full results of the X-ray analysis will be presented by (Komossa et al. 2012, in prep.).

4.2. E1821+643. The well-known luminous quasar E1821+ $643(z=0.297)$ shows highly asymmetric broad Balmer lines which appear different in direct and in polarized light, and are strongly shifted with respect to the narrow lines. Based on their spectropolarimetric observations, Robinson et al. [74] favor a scenario where one component of the BLR is bound to a recoiling black hole, which is moving at $2100 \mathrm{~km} / \mathrm{s}$ relative to its host galaxy. A second broad-line system is shifted by only $470 \mathrm{~km} / \mathrm{s}$, and its nature is currently unclear. If still related to recoil, in form of a marginally bound or unbound component of the BLR, the system is young, and Robinson et al. then estimate an age of $\sim 10^{4}$ years.

4.3. SDSSJ105041.35+345631.3. Shields et al. [68, 75] selected the quasar SDSSJ105041.35+345631.3 at $z=0.272$ from the SDSS because of its large kinematic shift of the BLR of, in this case, $3500 \mathrm{~km} / \mathrm{s}$ relative to the narrow emission lines. A projection effect is considered unlikely, as is a binary SMBH because of the lack of detectable orbital motion. While Shields et al. do not rule out an extreme case of a recoiling $\mathrm{SMBH}$, they conclude that several aspects of the optical spectrum are best understood if this galaxy is an extreme case of a "double-peaked emitter."

\section{Candidate Recoiling SMBHs Identified by Spatial Offsets}

5.1. CID-42. The galaxy CID-42 (COSMOSJ1000+0206) at redshift $z=0.359$ was discovered in the COSMOS survey [76], and caught attention due to its unusual morphology with two apparent optical "nuclei" [77] at a projected separation of $2 \mathrm{kpc}$, and an extended tidal tail. Initially suspected to be a binary AGN [77], it was then reinterpreted as a candidate recoiling $\mathrm{SMBH}$, or alternatively, an $\mathrm{SMBH}$ ejection following 3-body interaction in a triple $\mathrm{SMBH}$ system, by Civano et al. [78]. An HST image analysis has shown that the northwestern core is slightly extended though compact, and consistent with being the nucleus of the galaxy, while only the southeastern bright source is point-like [78]. The optical spectrum of CID 42 shows a kinematic shift of $1200 \mathrm{~km} / \mathrm{s}$ between the BLR and the major narrow-line component, and extra faint narrow $\mathrm{H} \beta$ emission at the same redshift as the broad lines [78], and perhaps further faint narrow-line emission shifted by $\sim 150 \mathrm{~km} / \mathrm{s}$ [77]. As such, the spectrum shares similarities with that of the recoil candidate SDSSJ092712.65+294344.0 [34, 68]. Another remarkable feature, not yet well understood, is the presence of a strong redshifted broad iron line with a P-Cygni profile, variable over four years, of high column density and highly ionized [78]. Follow-up optical and X-ray observations are currently underway (Civano et al. 2012, in prep.). Their new highresolution Chandra data show the presence of only one $\mathrm{X}$ ray emitting object which coincides with the position of the southeastern optical source, supporting the recoil scenario rather than the binary SMBH (Civano et al. 2012, in prep.).

5.2. M87. M87 is a nearby massive galaxy with a prominent radio jet. The photocenter of the host galaxy is offset by $7 \mathrm{pc}$ from the nuclear point source (i.e., presumably the location of the SMBH) [79]. The displacement is in the direction of the counterjet. Among several scenarios (acceleration by a jet, presence of massive perturbers, binary orbital motion) considered, Batcheldor et al. [79] favor GW recoil as the most plausible. The observed offset can then be explained either by a moderate kick 1 Myr ago, or residual small-amplitude oscillations of a large recoil which happened $<1$ Gyr ago.

5.3. CXOJ122518.6+144545. Jonker et al. [80] reported the detection of an unusual off-nuclear X-ray source, at a projected separation of $3 \mathrm{kpc}$ from the core of the galaxy SDSSJ122518.86+144547.7 at $z=0.045$. CXOJ122$518.6+144545$ is X-ray luminous and has a bright optical counterpart and properties unlike those of other off-nuclear X-ray sources which were found in large numbers with Chandra. The authors offer three explanations of CXOJ122518.6+144545: a supernova of type IIn, an ultraluminous $\mathrm{X}$-ray source with an unusually bright optical counterpart, or a recoiling SMBH. Bellovary et al. [81] further discuss the possibility of a wandering $\mathrm{SMBH}$ in the galaxy halo, produced by stripping of a satellite which merged with the primary galaxy.

5.4. ESO 1327-2041. The nearby galaxy ESO 1327-2041 $(z=$ 0.018 ) shows a complex morphology indicative of a recent merger. HST imaging has revealed the presence of a compact source embedded in an extended "stellar stream" ([82]; their Figure 1), at a redshift similar to the core of ESO 1327-2041, and at a projected separation of $15 \mathrm{kpc}$. Keeney et al. discuss several possible interpretations of this compact object, and propose that it is the actual nucleus of the galaxy, ejected as a consequence of either tidal interaction between two galaxies or gravitational wave recoil following a past merger.

\section{Implications of Recoil Oscillations for Unified Models of AGN}

There are potentially far-reaching consequences of $\mathrm{SMBH}$ recoil for unified models of AGN. Spatial oscillations of the SMBHs about the cores of their host galaxies imply that the SMBHs spend a significant fraction of time off-nucleus, at scales beyond that of the molecular obscuring torus. An intrinsically obscured quasar of type 2 with its BLR hidden by the torus will therefore appear as unabsorbed, type 1 quasar during the recoil oscillations, when moving beyond the torus scale.

Assuming reasonable distributions of recoil velocities, Komossa and Merritt [23] have computed the off-core timescale of (intrinsically type 2) quasars (These calculations are based on models of Gualandris and Merritt [22], which did not include gas. Recent simulations of recoil oscillations in 
a gaseous disk show, that oscillation timescales can either increase or decrease [83] with respect to the gas-free case.). It was shown that roughly $50 \%$ of all major mergers result in a $\mathrm{SMBH}$ being displaced beyond the torus for a time of $10^{7.5} \mathrm{yr}$ or more. This is an interesting number, because it is comparable to quasar activity time scales. Since major mergers (i.e., quasars) are most strongly affected by gravitational wave recoil, our results imply a deficiency of luminous type 2 quasars in comparison to low-luminosity Seyfert 2 galaxies, as indeed observed (e.g., [84]). These may therefore naturally explain the long-standing puzzle, why few absorbed type 2 quasars exist at high luminosities; it would be these which are affected by the recoil oscillations, therefore appearing as type 1 rather than type 2 for a significant fraction of their lifetime [23].

Recoil oscillations further imply the presence of a fraction of quasars which lack a hot dust component, because the dusty torus is only illuminated from a distance. Such "hotdust-poor" quasars have indeed been observed (e.g., [85]).

Recoil oscillations also have a number of other observable consequences related to AGN. For instance, they will affect the X-ray background and its modeling since a fraction of sources will be unobscured at any given time. In particular, small amplitude oscillations of the order the torus size will affect the ratio of Compton-thin to Compton-thick sources and could lead to measurable variability in the absorption and extinction of AGN spectra once the recoiling SMBH passes the individual clouds making up the torus [23].

\section{Astrophysical Implications and Future Observations}

The kicks and superkicks predicted by recent numerical relativity simulations of coalescing SMBHs have stimulated an active new field of research. Electromagnetic signatures of recoiling SMBHs are being predicted, several candidates have emerged in large data bases, and astrophysical implications of the kicks are still being explored. The fact that SMBHs will not always reside at the very cores of their host galaxies, or may even be ejected completely, has many potential implications for the topics discussed in this book; for galaxy and $\mathrm{SMBH}$ assembly and galaxy-SMBH (co)evolution, core structures in early type galaxies, the scatter in the host galaxySMBH scaling relations, the statistics of obscured quasars, and the redshift-dependence of gravitational wave signals (e.g., $[26,86-92])$.

It is therefore important to identify more candidate recoiling SMBHs through observations. Promising future searches would include (1) emission-line signatures in large spectroscopic data bases such as SDSS or LAMOST; (2) recoil flares from accretion disks and stellar tidal disruptions in large-scale surveys like Pan-STARRS and LSST and in the Xray regime; (3) the characteristic, large stellar velocity dispersions of HCSSs in spectroscopic followups of ongoing imaging surveys of nearby clusters of galaxies.

Detecting recoiling SMBHs in large numbers will open up a new window on measuring galaxy merger histories and kick amplitude distributions, and testing predictions of numerical relativity.

\section{References}

[1] M. C. Begelman, R. D. Blandford, and M. J. Rees, "Massive black hole binaries in active galactic nuclei," Nature, vol. 287, no. 5780, pp. 307-309, 1980.

[2] S. Komossa, "Observational evidence for binary black holes and active double nuclei," Memorie della Società Astronomica Italiana, vol. 77, p. 733, 2006.

[3] J.D. Bekenstein, "Gravitational-radiation recoil and runaway black holes," The Astrophysical Journal, vol. 183, pp. 657-664, 1973.

[4] M. Campanelli, C. Lousto, Y. Zlochower, and D. Merritt, "Large merger recoils and spin flips from generic black hole binaries," The Astrophysical Journal, vol. 659, no. 1, pp. L5-L8, 2007.

[5] M. Campanelli, C. O. Lousto, H. Nakano, and Y. Zlochower, "Comparison of numerical and post-Newtonian waveforms for generic precessing black-hole binaries," Physical Review D, vol. 79, no. 8, Article ID 084010, 2009.

[6] J. A. González, M. Hannam, U. Sperhake, B. Brügmann, and S. Husa, "Supermassive recoil velocities for binary black-hole mergers with antialigned spins," Physical Review Letters, vol. 98, no. 23, Article ID 231101, 2007.

[7] J. A. González, U. Sperhake, and B. Brügmann, "Black-hole binary simulations: the mass ratio $10: 1$, Physical Review D, vol. 79, no. 12, Article ID 124006, 2009.

[8] F. Herrmann, I. Hinder, D. M. Shoemaker, P. Laguna, and R. A. Matzner, "Binary black holes: spin dynamics and gravitational recoil," Physical Review D, vol. 76, no. 8, Article ID 084032, 2007.

[9] J. G. Baker, W. D. Boggs, J. Centrella et al., "Modeling kicks from the merger of generic black hole binaries," The Astrophysical Journal, vol. 682, no. 1, pp. L29-L32, 2008.

[10] B. Brügmann, J. A. González, M. Hannam, S. Husa, and U. Sperhake, "Exploring black hole superkicks," Physical Review D, vol. 77, no. 12, Article ID 124047, 2008.

[11] S. Dain, C. O. Lousto, and Y. Zlochower, "Extra-large remnant recoil velocities and spins from near-extremal-Bowen- Yorkspin black-hole binaries," Physical Review D, vol. 78, no. 2, Article ID 024039, 2008.

[12] S. H. Miller and R. A. Matzner, "Multipole analysis of kicks in collision of spinning binary black holes," General Relativity and Gravitation, vol. 41, no. 3, pp. 525-539, 2009.

[13] C. O. Lousto and Y. Zlochower, "Modeling gravitational recoil from precessing highly spinning unequal-mass black-hole binaries," Physical Review D, vol. 79, no. 6, Article ID 064018, 2009.

[14] A. Le Tiec, L. Blanchet, and C. M. Will, "The gravitationalwave recoil from the ringdown phase of coalescing black hole binaries," Classical and Quantum Gravity, vol. 27, no. 1, Article ID 012001, 2010.

[15] C. O. Lousto, M. Campanelli, Y. Zlochower, and H. Nakano, "Remnant masses, spins and recoils from the merger of generic black hole binaries," Classical and Quantum Gravity, vol. 27, no. 11, Article ID 114006, 2010.

[16] C. O. Lousto and Y. Zlochower, "Modeling maximum astrophysical gravitational recoil velocities," Physical Review D, vol. 83, no. 2, 2011.

[17] J. Centrella, J. G. Baker, B. J. Kelly, and J. R. van Meter, "Blackhole binaries, gravitational waves, and numerical relativity," Reviews of Modern Physics, vol. 82, no. 4, pp. 3069-3119, 2010.

[18] C. Lousto and Y. Zlochower, "Hangup kicks: still larger recoils by partial spin/orbit alignment of black-hole binaries," Physical Review Letters, vol. 107, Article ID 231102, 2011. 
[19] J. Healy, F. Herrmann, I. Hinder, D. M. Shoemaker, P. Laguna, and R. A. Matzner, "Superkicks in hyperbolic encounters of binary black holes," Physical Review Letters, vol. 102, no. 4, Article ID 041101, 2009.

[20] U. Sperhake, E. Berti, V. Cardoso, F. Pretorius, and N. Yunes, "Superkicks in ultrarelativistic encounters of spinning black holes," Physical Review D, vol. 83, no. 2, Article ID 024037, 2011.

[21] P. Madau and E. Quataert, "The effect of gravitational-wave recoil on the demography of massive black holes," The Astrophysical Journal, vol. 606, no. 1, pp. L17-L20, 2004.

[22] A. Gualandris and D. Merritt, "Ejection of supermassive black holes from galaxy cores," The Astrophysical Journal, vol. 678, no. 2, pp. 780-797, 2008.

[23] S. Komossa and D. Merritt, "Gravitational wave recoil oscillations of black holes: implications for unified models of active galactic nuclei," The Astrophysical Journal, vol. 689, no. 2, pp. L89-L92, 2008.

[24] P. Madau, M. J. Rees, M. Volonteri, F. Haardt, and S. P. Oh, "Early reionization by miniquasars," The Astrophysical Journal, vol. 604, no. 2, pp. 484-494, 2004.

[25] D. Merritt, M. Milosavljević, M. Favata, S. A. Hughes, and D. E. Holz, "Consequences of gravitational radiation recoil," The Astrophysical Journal, vol. 607, no. 1, pp. L9-L12, 2004.

[26] N. I. Libeskind, S. Cole, C. S. Frenk, and J. C. Helly, “The effect of gravitational recoil on black holes forming in a hierarchical universe," Monthly Notices of the Royal Astronomical Society, vol. 368, no. 3, pp. 1381-1391, 2006.

[27] A. Loeb, "Observable signatures of a black hole ejected by gravitational-radiation recoil in a Galaxy merger," Physical Review Letters, vol. 99, no. 4, Article ID 041103, 2007.

[28] L. Blecha and A. Loeb, "Effects of gravitational-wave recoil on the dynamics and growth of supermassive black holes," Monthly Notices of the Royal Astronomical Society, vol. 390, no. 4, pp. 1311-1325, 2008.

[29] M. Volonteri and P. Madau, "Off-nuclear AGNs as a signature of recoiling massive black holes," The Astrophysical Journal, vol. 687, no. 2, Article ID 10.1086/593353, p. L57, 2008.

[30] F. K. Liu, D. Wang, and X. Chen, "Recoiling supermassive black holes in spin-flip radio galaxies," The Astrophysical Journal, http://arxiv.org/abs/1112.1081.

[31] D. Merritt, T. Storchi-Bergmann, A. Robinson, D. Batcheldor, D. Axon, and R. C. Fernandes, "The nature of the HE04502958 system," Monthly Notices of the Royal Astronomical Society, vol. 367, no. 4, pp. 1746-1750, 2006.

[32] B. M. Peterson, "The masses of black holes in active galactic nuclei," in Proceedings of the Central Engine of Active Galactic Nuclei, vol. 373 of ASP Conference Series, p. 3, Jioatong University, October 2006.

[33] E. W. Bonning, G. A. Shields, and S. Savliander, "Recoiling black holes in quasars," The Astrophysical Journal, vol. 666, no. 1, pp. L13-L16, 2007.

[34] S. Komossa, H. Zhou, and H. Lu, "A recoiling supermassive black hole in the quasar SDSS J092712.65+294344.0?" The Astrophysical Journal, vol. 678, no. 2, pp. L81-L84, 2008.

[35] M. Eracleous, T. A. Boroson, J. P. Halpern, and J. Liu, "A large systematic search for recoiling and close supermassive binary black holes," http://arxiv.org/abs/1106.2952.

[36] F. K. Liu, X. B. Wu, and S. L. Cao, "Double-double radio galaxies: remnants of merged supermassive binary black holes," Monthly Notices of the Royal Astronomical Society, vol. 340, no. 2, pp. 411-416, 2003.
[37] M. Milosavljević and E. S. Phinney, "The afterglow of massive black hole coalescence," The Astrophysical Journal, vol. 622, no. 2, pp. L93-L96, 2005.

[38] M. Ponce, J.A. Faber, and J.C. Lombardi, "Accretion disks around kicked black holes: post-kick dynamics," The Astrophysical Journal, http://arxiv.org/abs/1107.1711, vol. 745, p. 71, 2012.

[39] Z. Lippai, Z. Frei, and Z. Haiman, "Prompt shocks in the gas disk around a recoiling supermassive black hole binary," The Astrophysical Journal Letters, vol. 676, no. 1, pp. L5-L8, 2008.

[40] G. A. Shields and E. W. Bonning, "Powerful flares from recoiling black holes in quasars," The Astrophysical Journal, vol. 682, no. 2, pp. 758-766, 2008.

[41] J. D. Schnittman and J. H. Krolik, "The infrared afterglow of supermassive black hole mergers," The Astrophysical Journal, vol. 684, no. 2, pp. 835-844, 2008.

[42] M. Megevand, M. Anderson, J. Frank et al., "Perturbed disks get shocked: Binary black hole merger effects on accretion disks," Physical Review D, vol. 80, no. 2, Article ID 024012, 2009.

[43] E. M. Rossi, G. Lodato, P. J. Armitage, J. E. Pringle, and A. R. King, "Black hole mergers: the first light," Monthly Notices of the Royal Astronomical Society, vol. 401, no. 3, pp. 2021-2035, 2010.

[44] L. R. Corrales, Z. Haiman, and A. MacFadyen, "Hydrodynamical response of a circumbinary gas disc to black hole recoil and mass loss," Monthly Notices of the Royal Astronomical Society, vol. 404, no. 2, pp. 947-962, 2010.

[45] T. Tanaka, Z. Haiman, and K. Menou, "Witnessing the birth of a quasar," Astronomical Journal, vol. 140, no. 2, pp. 642-651, 2010.

[46] O. Zanotti, C. Roedig, L. Rezzolla, and L. del Zanna, "General relativistic radiation hydrodynamics of accretion flows-I. Bondi-Hoyle accretion," Monthly Notices of the Royal Astronomical Society, vol. 417, no. 4, pp. 2899-2915, 2011.

[47] S. Komossa and N. Bade, "The giant X-ray outbursts in NGC 5905 and IC 3599: follow-up observations and outburst scenarios," Astronomy and Astrophysics, vol. 343, no. 3, pp. 775787, 1999.

[48] J. S. Bloom, D. Giannios, B. D. Metzger et al., "A possible relativistic jetted outburst from a massive black hole fed by a tidally disrupted star," Science, vol. 333, no. 6039, pp. 203-206, 2011.

[49] S. Komossa and D. Merritt, "Tidal disruption flares from recoiling supermassive black holes," The Astrophysical Journal, vol. 683, no. 1, pp. L21-L24, 2008.

[50] N. Stone and A. Loeb, "Prompt tidal disruption of stars as an electromagnetic signature of supermassive black hole coalescence," Monthly Notices of the Royal Astronomical Society, vol. 412, no. 1, pp. 75-80, 2011.

[51] X. Chen, P. Madau, A. Sesana, and F. K. Liu, "Enhanced tidal disruption rates from massive black hole binaries," The Astrophysical Journal, vol. 697, no. 2, pp. L149-L152, 2009.

[52] D. Merritt, J. D. Schnittman, and S. Komossa, "Hypercompact stellar systems around recoiling supermassive black holes," The Astrophysical Journal, vol. 699, no. 2, pp. 1690-1710, 2009.

[53] R. M. O'Leary and A. Loeb, “Star clusters around recoiled black holes in the Milky Way halo," Monthly Notices of the Royal Astronomical Society, vol. 395, no. 2, pp. 781-786, 2009.

[54] K. Holley-Bockelmann, K. Gültekin, D. Shoemaker, and N. Yunes, "Gravitational wave recoil and the retention of intermediate-mass black holes," The Astrophysical Journal, vol. 686, no. 2, pp. 829-837, 2008. 
[55] R. M. O'Leary and A. Loeb, "Recoiled star clusters in the Milky Way halo: N-body simulations and a candidate search through SDSS," http://arxiv.org/abs/1102.3695.

[56] D. A. Kornreich and R. V. E. Lovelace, "Dynamics of kicked and accelerated massive black holes in galaxies," The Astrophysical Journal, vol. 681, no. 1, pp. 104-112, 2008.

[57] B. Devecchi, E. Rasia, M. Dotti, M. Volonteri, and M. Colpi, "Imprints of recoiling massive black holes on the hot gas of early-type galaxies," Monthly Notices of the Royal Astronomical Society, vol. 394, no. 2, pp. 633-640, 2009.

[58] Y. Fujita, "Long-term evolution of and X-ray emission from a recoiling supermassive black hole in a disk galaxy," The Astrophysical Journal, vol. 691, no. 2, pp. 1050-1057, 2009.

[59] R. De La Fuente Marcos and C. De La Fuente Marcos, "The invisible hand: star formation triggered by runaway black holes," The Astrophysical Journal, vol. 677, no. 1, pp. L47-L50, 2008.

[60] J. D. Schnittman and A. Buonanno, "The distribution of recoil velocities from merging black holes," The Astrophysical Journal, vol. 662, no. 2, pp. L63-L66, 2007.

[61] M. Kesden, U. Sperhake, and E. Berti, "Relativistic suppression of black hole recoils," The Astrophysical Journal, vol. 715, no. 2, pp. 1006-1011, 2010.

[62] M. Volonteri, M. Sikora, and J. P. Lasota, "Black hole spin and galactic morphology," The Astrophysical Journal, vol. 667, no. 2, pp. 704-713, 2007.

[63] P. A. G. Scheuer and R. Feiler, "The realignment of a black hole misaligned with its accretion disc," Monthly Notices of the Royal Astronomical Society, vol. 282, no. 1, pp. 291-294, 1996.

[64] P. Natarajan and P. J. Armitage, "Warped discs and the directional stability of jets in active galactic nuclei," Monthly Notices of the Royal Astronomical Society, vol. 309, no. 4, pp. 961-968, 1999.

[65] T. Bogdanović, C. S. Reynolds, and M. C. Miller, "Alignment of the spins of supermassive black holes prior to coalescence," The Astrophysical Journal, vol. 661, no. 2, pp. L147-L150, 2007.

[66] A. Perego, M. Dotti, M. Colpi, and M. Volonteri, "Mass and spin co-evolution during the alignment of a black hole in a warped accretion disc," Monthly Notices of the Royal Astronomical Society, vol. 399, no. 4, pp. 2249-2263, 2009.

[67] M. Dotti, M. Volonteri, A. Perego, M. Colpi, M. Ruszkowski, and F. Haardt, "Dual black holes in merger remnants-II. Spin evolution and gravitational recoil," Monthly Notices of the Royal Astronomical Society, vol. 402, no. 1, pp. 682-690, 2010.

[68] G. A. Shields, E. W. Bonning, and S. Salviander, "Comment on the black hole recoil candidate quasar SDSS J092712.65+294344.0," The Astrophysical Journal, vol. 696, no. 2, pp. 1367-1373, 2009.

[69] T. M. Heckman, J. H. Krolik, S. M. Moran, J. Schnittman, and S. Gezari, "SDSSJ092712.65+294344.0: NGC 1275 at $z=0.7$ ?" The Astrophysical Journal, vol. 695, no. 1, article 363, 2009.

[70] M. Dotti, C. Montuori, R. Decarli, M. Volonteri, M. Colpi, and F. Haardt, "SDSSJ092712.65+294344.0: a candidate massive black hole binary," Monthly Notices of the Royal Astronomical Society, vol. 398, no. 1, pp. L73-L77, 2009.

[71] T. Bogdanović, M. Eracleous, and S. Sigurdsson, "SDSS J092712.65+294344.0: recoiling black hole or a subparsec binary candidate?" The Astrophysical Journal, vol. 697, no. 1, pp. L288-L292, 2009.

[72] R. Decarli, M. T. Reynolds, and M. Dotti, "A photometric study of the field around the candidate recoiling/binary black hole SDSS J092712.65+294344.0," Monthly Notices of the Royal Astronomical Society, vol. 397, no. 1, pp. 458-466, 2009.
[73] M. Vivek, R. Srianand, P. Noterdaeme, V. Mohan, and V. C. Kuriakose, "SDSS J092712.64+294344.0: recoiling blackhole or merging galaxies?" Monthly Notices of the Royal Astronomical Society, vol. 400, no. 1, pp. L6-L9, 2009.

[74] A. Robinson, S. Young, D. J. Axon, P. Kharb, and J. E. Smith, "Spectropolarimetric evidence for a kicked supermassive black hole in the quasar E1821+643," The Astrophysical Journal, vol. 717, no. 2, pp. L122-L126, 2010.

[75] G. A. Shields, D. J. Rosario, K. L. Smith et al., "The quasar SDSS J105041.35+345631.3: black hole recoil or extreme double-peaked emitter?" The Astrophysical Journal, vol. 707, no. 2, pp. 936-941, 2009.

[76] M. Elvis, in Proceedings of the BAAS, vol. 41, p. 708, 2009.

[77] J. M. Comerford, R. L. Griffith, B. F. Gerke et al., "1.75 h -1 kpc separation dual active galactic nuclei at $\mathrm{z}=0.36$ in the cosmos field," The Astrophysical Journal, vol. 702, no. 1, pp. L82-L86, 2009.

[78] F. Civano, M. Elvis, G. Lanzuisi et al., "A runaway black hole in cosmos: gravitational wave or slingshot recoil?" The Astrophysical Journal, vol. 717, no. 1, pp. 209-222, 2010.

[79] D. Batcheldor, A. Robinson, D. J. Axon, E. S. Perlman, and D. Merritt, "A displaced supermassive black hole in M87," The Astrophysical Journal, vol. 717, pp. L6-L10, 2010.

[80] P. G. Jonker, M. A. P. Torres, A. C. Fabian, M. Heida, G. Miniutti, and D. Pooley, "A bright off-nuclear X-ray source: a type IIn supernova, a bright ULX or a recoiling supermassive black hole in CXO J122518.6+144545," Monthly Notices of the Royal Astronomical Society, vol. 407, no. 1, pp. 645-650, 2010.

[81] J. M. Bellovary, F. Governato, T. R. Quinn, J. Wadsley, S. Shen, and M. Volonteri, "Wandering black holes in bright disk galaxy halos," The Astrophysical Journal, vol. 721, no. 2, pp. L148-L152, 2010.

[82] B. A. Keeney, J. T. Stocke, C. W. Danforth, and C. L. Carilli, "The quasar/galaxy pair PKS 1327-206/ESO 1327-2041: absorption associated with a recent galaxy merger," Astronomical Journal, vol. 141, no. 2, article 66, 2011.

[83] D. Sijacki, V. Springel, and M. G. Haehnelt, "Gravitational recoils of supermassive black holes in hydrodynamical simulations of gas-rich galaxies," Monthly Notices of the Royal Astronomical Society, vol. 414, no. 4, pp. 3656-3670, 2011.

[84] G. Hasinger, "Absorption properties and evolution of active galactic nuclei," Astronomy and Astrophysics, vol. 490, no. 3, pp. 905-922, 2008.

[85] H. Hao, M. Elvis, F. Civano, and A. Lawrence, "Hot-dust-poor quasars in mid-infrared and optically selected samples," The Astrophysical Journal, vol. 733, no. 2, article 108, 2011.

[86] Z. Haiman, "Constraints from gravitational recoil on the growth of supermassive black holes at high redshift," The Astrophysical Journal, vol. 613, no. 1, pp. 36-40, 2004.

[87] M. Boylan-Kolchin, C. P. Ma, and E. Quataert, "Core formation in galactic nuclei due to recoiling black holes," The Astrophysical Journal, vol. 613, no. 1, pp. L37-L40, 2004.

[88] J. D. Schnittman and A. Buonanno, "The distribution of recoil velocities from merging black holes," The Astrophysical Journal, vol. 662, no. 2, pp. L63-L66, 2007.

[89] A. Sesana, "Extreme recoils: impact on the detection of gravitational waves from massive black hole binaries," Monthly Notices of the Royal Astronomical Society, vol. 382, no. 1, pp. L6-L10, 2007.

[90] T. Tanaka and Z. Haiman, "The assembly of supermassive black holes at high redshifts," The Astrophysical Journal, vol. 696, no. 2, pp. 1798-1822, 2009. 
[91] M. Volonteri, K. Gültekin, and M. Dotti, “Gravitational recoil: effects on massive black hole occupation fraction over cosmic time," Monthly Notices of the Royal Astronomical Society, vol. 404, no. 4, pp. 2143-2150, 2010.

[92] M. Micic, K. Holley-Bockelmann, and S. Sigurdsson, "Growing massive black holes in a Local Group environment: the central supermassive, slowly sinking and ejected populations," Monthly Notices of the Royal Astronomical Society, vol. 414, no. 2, pp. 1127-1144, 2011. 

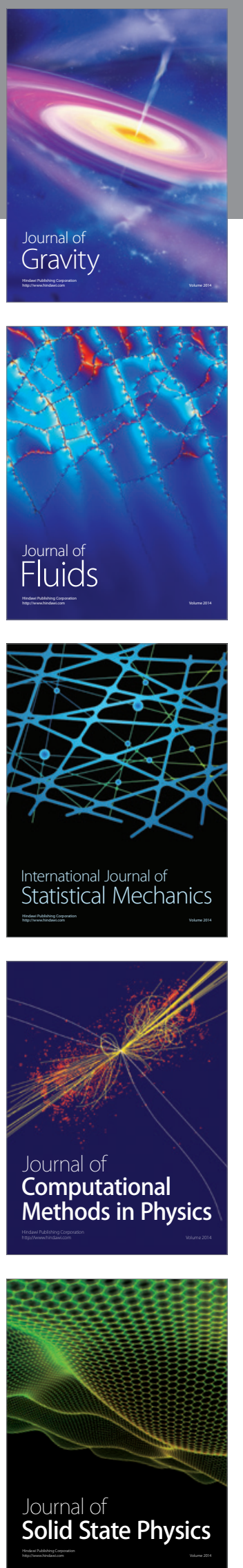

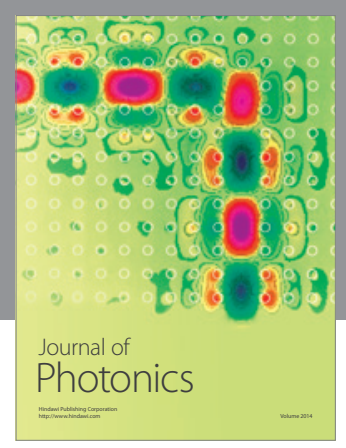

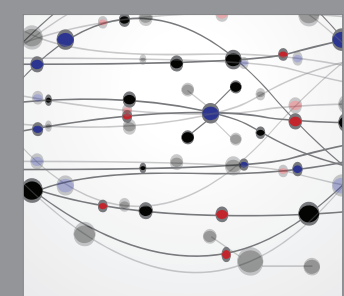

The Scientific World Journal
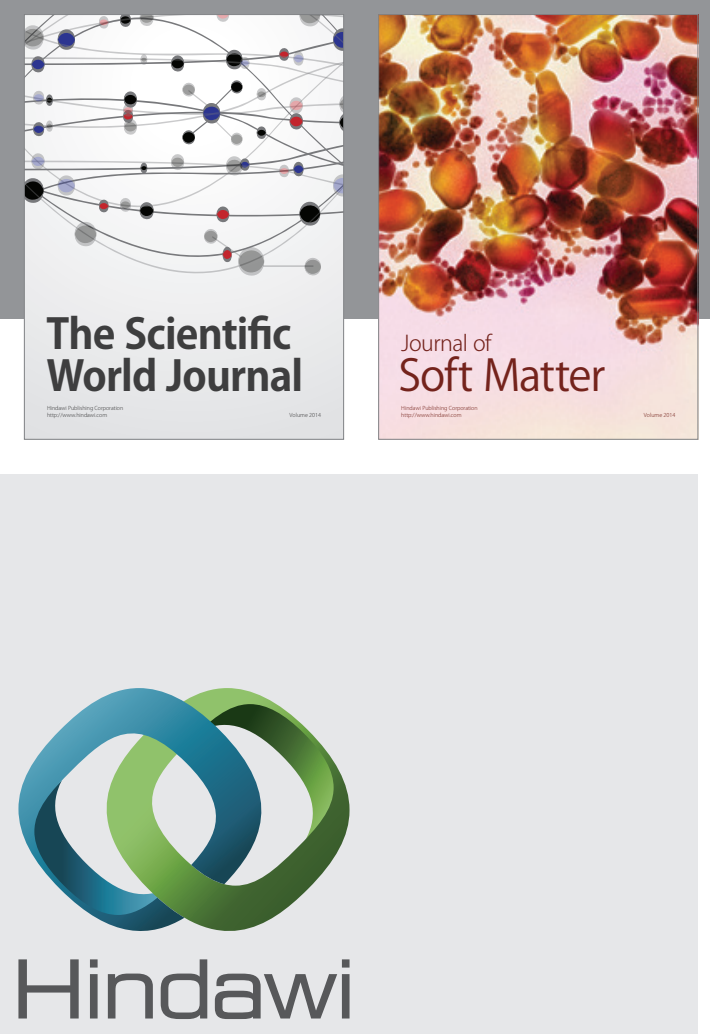

Submit your manuscripts at

http://www.hindawi.com
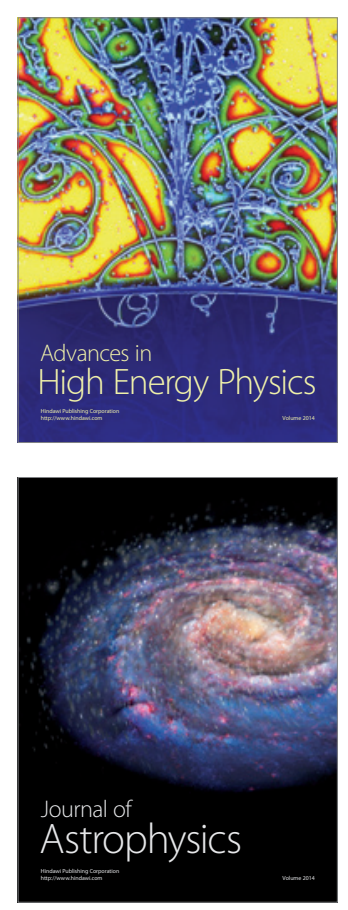
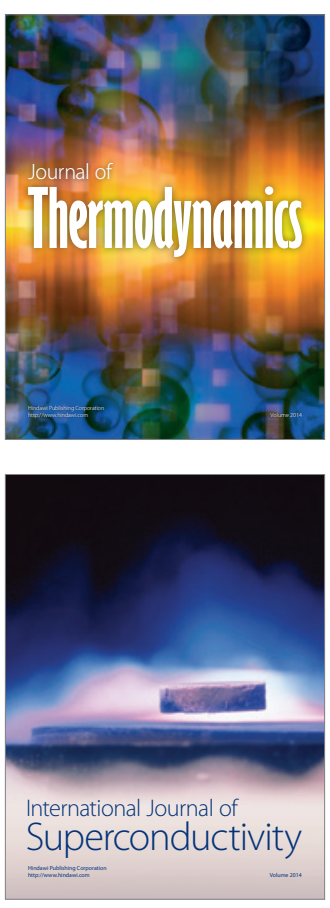
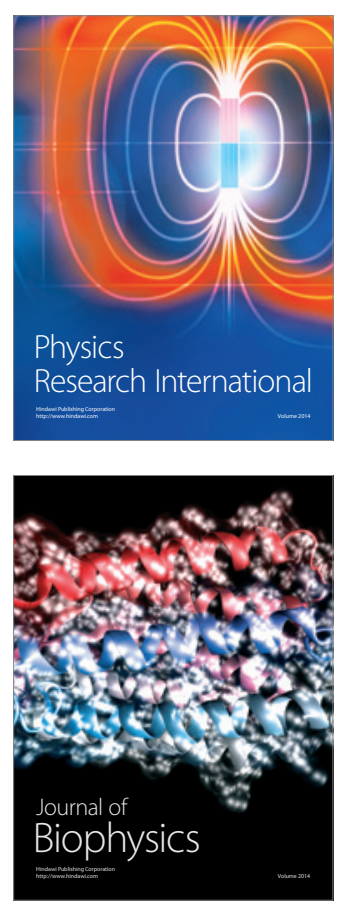
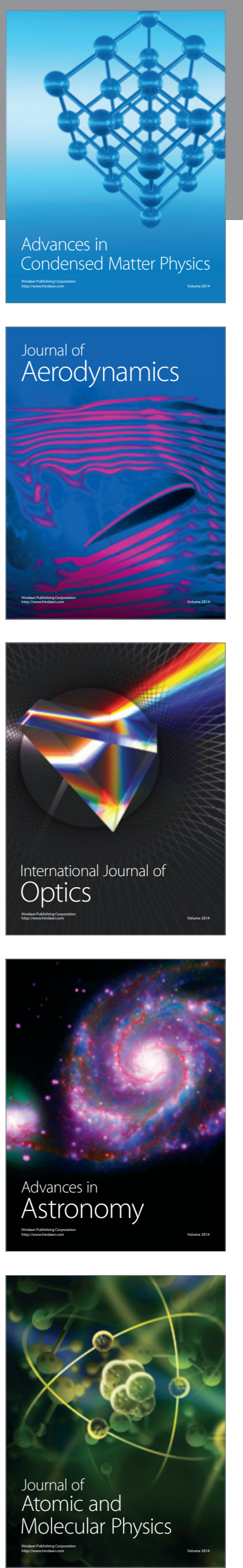